\title{
Trastorno específico del lenguaje y su implicación con los procesos predictores para el aprendizaje escolar. estrategias de intervención
}

\author{
Specific disorder of language and its involvement with predictive \\ processes for school learning. Intervention strategies.
}

Martinez-Martinez, Aura-Jazmín ${ }^{1}$; Caicedo-Tarazona, Ana-María ${ }^{2}$; Rodríguez-Quiñones, Daniela $^{3}$

Como citar este artículo: Martinez-Martinez, Aura-Jazmín; Caicedo-Tarazona, Ana-María; Rodríguez-Quiñones, Daniela. Trastorno específico del lenguaje y su implicación con los procesos predictores para el aprendizaje escolar. estrategias de intervención. Revistas Científica Signos Fónicos. 2020: 6 (2). 42-61.

Recibido: diciembre 11, 2019. Aprobado: marzo 23, 2020.

\section{RESUMEN}

INTRODUCCIÓN: el objetivo de este estudio fue establecer a través de una revisión sobre la evidencia científica, la cual permite establecer qué es el TEL y su implicación en los procesos predictores para el aprendizaje y a su vez desarrollar estrategias para intervenir. MÉTODOS: esta investigación tiene soporte metodológico mixto. Para la estructuración de las preguntas se utilizó la metodología PICO y GRADE, donde se inicia la revisión, con indicadores de producción del lenguaje asociadas al TEL y a sus procesos de intervención. RESULTADOS: arroja que por medio de la revisión se evidencia un referente teórico que nos indica el método más utilizado para la evaluación es el de los Modelos de Adams, seguidamente se realizaron estrategias de intervención para cada uno de los componentes del lenguaje en usuarios con características propias de un TEL. ANÁLISIS Y DISCUSIÓN: según la NIDCD el TEL es una condición que retrasa el lenguaje, y presenta características disfuncionales en la producción de actos locutivos e ilocutivos en los componentes del lenguaje, lo cual requiere de estrategias de rehabilitación, para brindarles a los infantes con TEL mejorías en su estructura lingüística. CONCLUSIONES: existen métodos de evaluación para usuarios con TEL que tienen como objetivo evaluar procesos como atención, concentración, memoria de trabajo, discriminación auditiva, repetición de palabras y pseudopalabras, y cohesión coherencia en el discurso. Creando Aprendizajes es una estrategia de intervención en infantes con TEL en edades de 3 a 7 años desde el abordaje de cada uno de los componentes del lenguaje.

\footnotetext{
${ }^{1}$ Fonoaudiologa, Esp. Pedagogía Universitaria Magister en Intervención en las dificultades en el Aprendizaje,auramz03@gmail.com, Orcid:0000-0002-7695-1369, Centro de Neurorehabilitacion Body Brain( CENER), Cúcuta - Colombia.

2,3Practricantes, ana.caicedo13n@gmail.com,danielarq08@gmail.com.
} 
Martínez, Caicedo, Rodríguez. Revista Científica Signos Fónicos, 2020, 6 (2): 42 - 61.

PALABRAS CLAVE: trastorno del desarrollo del lenguaje, aprendizaje escolar, evaluación, intervención, signos y síntomas.

\begin{abstract}
INTRODUCTION: the objective of this study is to establish through a review of scientific evidence, which allows us to establish what is the Specific Language Impairment (SLI) and its involvement in the predictive processes for school learning and in turn develop strategies for their intervention. METHODS: this research has a mixed methodological support. For the structuring of the questions, the PICO and GRADE methodology was used, where the bibliographic review was initiated, researching in databases on children's population, with indicators of language production associated with the SLI and its intervention processes. RESULTS: this research shows that through the bibliographic review a theoretical reference is shown that indicates the most used method for the evaluation is that of the Adams Models, with contributions from Freeman and Dake, Schelstraete based on the MEHRIT model, followed by They carried out intervention strategies for each of the language components in users with characteristics of a SLI. ANALYSIS AND DISCUSSION: according to the NIDCD, the SLI is a condition that delays the language, and has dysfunctional characteristics in the production of locutionary and illocutionary acts in the language components, which requires rehabilitation strategies, to provide infants with SLI with improvements in their structure linguistics. CONCLUSIONS: it is claimed that there are various evaluation methods for users with SLI that aim to evaluate processes such as attention, concentration, working memory, production of vowel and consonant phonemes, auditory discrimination, syllabic segmentation, repetition of words and pseudowords, and cohesion coherence in speech. Creating learings is a strategy aimed at the intervention of infants with SLI in ages ranging from three for seven years of age from the approach of each of the language components.
\end{abstract}

KEYWORDS Language Development Disorders, Learning, Evaluation, Intervention, Signs and Symptoms.

\title{
INTRODUCCIÓN
}

La comunicación es donde se articula el lenguaje con sus diferentes niveles, semántico, sintáctico, fonético fonológico y pragmático, como resultado de esta integración, se da el desarrollo integral del proceso de emisión, el cual le permite al ser humano establecer un contacto lingüístico con el contexto a través de los diferentes actos de habla. Este intercambio con el medio, favorece la evolución del infante como ser biopsicosocial. El proceso de adquisición del aprendizaje escolar, valorando lo anterior cuando surgen alteraciones en alguno de esos aspectos que conforman el lenguaje pueden surgir diversos trastornos que perturban el desempeño global de las características funcionales del acto de habla.

Entre esas manifestaciones que afecta la locución, los lineamientos teóricos describen el trastorno específico del Lenguaje como una condición que retrasa la adquisición, comprensión, expresión y la praxis del lenguaje hablado o escrito. Los usuarios que manifiestan esta situación evidencian unos indicadores anómalos de producción de las funciones del lenguaje por debajo de su edad cronológica, no identifican el significado y el significante, problemas de procesamiento o abstracción de la información (1),(2),(3),(4),(5); falencias en la estructuración de oraciones conformadas por artículos verbo, sustantivo, adjetivos calificativos. Igualmente, en el momento de soportar una conversación carecen de las habilidades propias de la pragmática tales como iniciar, mantener, finalizar un intercambio con sus homólogos, es necesario resaltar que estos infantes no presentan una patología de 
Martínez, Caicedo, Rodríguez. Revista Científica Signos Fónicos, 2020, 6 (2): 42 - 61.

origen orgánico, tales como pérdidas auditivas, deficiencias cognitivas, alteraciones del sistema nervioso central, entre otros.

En miras a brindarles estrategias que permitan beneficiar la calidad de vida a estos usuarios, y en pro a proporcionarles a los terapeutas que intervienen de forma integral, en especial al Fonoaudiólogo métodos de rehabilitación que le permitan admitir un buen pronóstico con respecto a la evolución del trastorno (6),(7),(8),(9),(10),(11); es necesario recurrir a la academia para enlazar a través de la investigación el nivel de evidencia científica, para darle valor de uso a lo anterior en el presente trabajo se realizó una búsqueda exhaustiva de los lineamientos teóricos de los diversos modelos de rehabilitación de los componentes del lenguaje, teniendo en cuenta estos aspectos se origina los siguientes interrogantes ¿Cuál es el método más utilizado para la evaluación en infantes Trastorno Específico del Lenguaje? Y ¿Qué estrategias terapéuticas a nivel de fonoaudiología se pueden implementar para niños con trastorno en el desarrollo del lenguaje?

\section{MÉTODOS}

Para el desarrollo de la presente investigación se emplea una metodológica de revisión sistemática de corte mixto en estudios primarios, con el objetivo de resumir la información existente respecto de un tema en particular. Los investigadores luego de recolectar los artículos de interés; los analizan, y comparan la evidencia que aportan con la de otros similares (12).

Dicha revisión se hará con una línea de tiempo de 10 años, que permite realizar una búsqueda, en las diferentes bases de datos (Pubmed, Redalyc, Scielo, Elsevier, Science Direct, AJSLP, RIE, UNED, Revista de psiquiatría infanto-juvenil, Neurología, Taylor \& Francis y Europe PMC) de los lineamientos teóricos que tienen en cuenta diversos enfoques en métodos de evaluación e intervención en infantes que presentan Trastornos Específicos del Lenguaje, con un compromiso a nivel de los componentes del lenguaje.

Esta recolección, organización y clasificación del respaldo teórico se dio gracias a la actividad practica de revisión sistemática. Dicha revisión se hará con la metodología PICO, la cual constituye un formato especializado para desarrollar la pregunta clínica.

Esta estrategia puede ser utilizada para construir diversos tipos de preguntas de investigación originadas de la práctica clínica, la formulación de preguntas con esta metodología sirve para mejorar la especificidad y claridad conceptual de los problemas clínicos a estudiar, así como para realizar búsquedas que arrojen resultados con mayor calidad y precisión.

Asimismo, permite maximizar la localización de información relevante, focalizando los objetivos de la búsqueda y rechazando la información innecesaria, lo cual ayudará a tomar las mejores decisiones basadas en la evidencia (13), (14), (15). Se debe tener en cuenta el significado de cada una de sus cifras, las cuales son:

P: Tipo de paciente (edad, sexo, estadio de la enfermedad, ámbito donde se realiza la atención, comorbilidad...) o problema de salud.

I: Intervención (factor pronóstico, agente etiológico, prueba diagnóstica, tratamiento...). 
Martínez, Caicedo, Rodríguez. Revista Científica Signos Fónicos, 2020, 6 (2): 42 - 61.

C: Alternativa a la intervención que queremos valorar (ausencia de un factor de riesgo, agente etiológico, patrón oro de una prueba diagnóstica...).

O: $($ outcomes $=$ resultados): Qué esperamos obtener como resultado de nuestra intervención (3).

En la Tabla 1 y 2 se observan las palabras claves consideradas para la evaluación e intervención respectivamente en el Trastorno Específico del Lenguaje con la metodología PICO.

TABLA 1. Categoría para método PICO.

\begin{tabular}{cccc}
\hline $\mathbf{P}$ & $\mathbf{I}$ & $\mathbf{C}$ & $\mathbf{O}$ \\
\hline $\begin{array}{c}\text { Trastorno Específico } \\
\text { del Lenguaje en } \\
\text { infantes. }\end{array}$ & Evaluación. & Eficacia. & Métodos. \\
\hline
\end{tabular}

Fuente: autores.

Tabla 2. Categoría para método PICO.

\begin{tabular}{cccc}
\hline P & I & C & O \\
\hline $\begin{array}{c}\text { Trastorno Específico } \\
\text { del Lenguaje en } \\
\text { infantes. }\end{array}$ & Intervención. & Rehabilitación. & Estrategias. \\
\hline
\end{tabular}

Fuente: autores.

Se analizaron sesenta artículos, los cuales se extrajeron de las bases de datos científica Pubmed, Redalyc, Scielo, Elsevier, Science Direct, AJSLP, RIE, UNED, Revista de psiquiatría infanto-juvenil, Neurología, Taylor \& Francis y Europe PMC, de los cuales cincuenta y dos cumplen con los criterios de inclusión establecidos previamente para ser base teórica de la respuesta a las preguntas problema.

Los criterios de inclusión establecidos fueron:

- Se dispone de una ventana de tiempo de diez años.

- Métodos de evaluación en el Trastorno Específico del Lenguaje.

- Métodos de intervención en el Trastorno Específico del Lenguaje.

- Bases de datos científicas pertenecientes a Pubmed, Redalyc, Scielo, Elsevier, Science Direct, AJSLP, RIE, UNED, Revista de psiquiatría infanto-juvenil, Neurología, Taylor \& Francis y Europe PMC.

Los criterios de exclusión de la información fueron:

- Artículos que no contemplen la evaluación del Trastorno Específico del Lenguaje.

- Artículos que no contemplen la intervención del Trastorno Específico del Lenguaje.

- Artículos o revistas que no cumplan con la venta de tiempo determinada.

- Artículos que no pertenezcan a las bases de datos establecidas anteriormente.

- Usuarios con otro tipo de alteración como alteraciones de audición o discapacidad cognoscitiva.

\section{RESULTADOS}

Por medio de la búsqueda en las bases de datos se encontraron una cantidad específica de artículos, de acuerdo con las palabras claves establecidas. Se hallaron un número de publicaciones las cuales fueron distribuidas en la Tabla 3.

Revista Científica Signos Fónicos, 2020,6(2): 1-21. ISNN 2422-1716. 
Martínez, Caicedo, Rodríguez. Revista Científica Signos Fónicos, 2020, 6 (2): 42 - 61.

Tabla 3. Resultados de la búsqueda en las bases de datos.

\begin{tabular}{cc}
\hline Base De Datos & Números de Estudios \\
\hline Redalyc & 16 \\
\hline Scielo & 9 \\
\hline Elsevier & 10 \\
\hline Science Direct & 7 \\
\hline Pubmed & 4 \\
\hline AJSLP & 2 \\
\hline UNED & 3 \\
\hline Revista de psiquiatría infanto- & 2 \\
\hline juvenil & 2 \\
\hline Neurología & 1 \\
\hline RIE & 3 \\
\hline Taylor \& Francis & 1 \\
\hline Europe PMC & $\mathbf{6 0}$
\end{tabular}

Fuente: Autores.

Se hallaron sesenta artículos en total, posteriormente se aplicaron los criterios de inclusión y exclusión, dejando así, cincuenta y dos artículos puesto que aportan información relevante en cuanto a los métodos para la evaluación e intervención en los Trastornos Específicos del Lenguaje. La misma información arrojó que existen diversos métodos de evaluación a usuarios con TEL, como lo son WISC-IV (escala de inteligencia de WECHSLER para niños-IV que abarca edades de 6 a 16 años, prueba del desarrollo fonológico de Laura Bosch de 3 a 7 años, escalas CELF (CELF 4, CELF Preeschool), ITPA (I, II, III), Escala de Desarrollo Cognitivo General, Prueba de Lenguaje PLS-4, Prueba del desarrollo inicial del lenguaje-Español y de los Modelos de Adams, con aportes de Freeman y Dake, Schelstraete con bases en el modelo MEHRIT, siendo este último el protocolo más eficaz para la evaluación según el referente teórico, donde recoge Adams 3 contextos que considera necesarios al momento de aplicar cualquiera de los protocolos mencionados anteriormente (4),(16),(17),(18),(19),(20).

El modelo social parte de la idea de que las dificultades pragmáticas surgen de una discapacidad en la intersubjetividad, es decir, en la capacidad de atribuir intención al comportamiento ajeno. Ese modelo propone, por lo tanto, reforzar la interacción social lo más pronto posible para enganchar al niño en los procesos de inferencia social. Mientras que el modelo cognitivo atribuye las dificultades pragmáticas a un déficit en la cognición social, es decir, en la capacidad de procesar adecuadamente la información proporcionada por el entorno, lo que constituye una actividad constructiva contextualizada.

Propone trabajar sobre la capacidad en establecer relaciones e inferencias y aprender las claves que permiten decodificar el comportamiento ajeno (4),(21),(22),(23),(24),(25). El modelo lingüístico considera que las dificultades pragmáticas son fundamentalmente consecuencia de un fallo en una competencia que los niños de desarrollo típico adquieren por observación, imitación y procesos de ensayo y error (5), (12).

Es habitual que estos programas incluyan también actividades para habilidades discursivas, consideradas como parte de las habilidades pragmáticas, como en el programa de estimulación del lenguaje para niños con TEA de Freeman, Dake y Schelstraete, en su revisión, llega a una clasificación bastante similar y recoge tipos de modelos: Los modelos centrados en la mejoría de la comunicación 
Martínez, Caicedo, Rodríguez. Revista Científica Signos Fónicos, 2020, 6 (2): 42 - 61.

social, por ejemplo, el social communication intervention program de Adams o el modelo MEHRIT (Casenhiser, Shanker y Stieben) que incluye trabajo en pequeños grupos, van dirigidos generalmente, pero no de manera exclusiva, a sujetos que presentan un cuadro de TEA y, en su mayoría, se basan en principios conductistas o cognitivo-conductistas (4),(26),(27),(28).

Los modelos de enfoque naturalístico enfocados al entorno familiar (como el programa Hanen, Sussman) o escolar (Mackay y Anderson) tienen un objetivo esencialmente preventivo. Los modelos centrados directamente en competencias pragmáticas: responden a principios de intervención directa y explícita en los que se explica y se entrena un contenido pragmático puntual a través de situaciones dirigidas, de role-playing o mediante soporte gráfico (12),(29),(30),(31),(32).

Luego de la revisión sistemática perteneciente a la intervención en usuarios con trastorno específico del Lenguaje se realizaron una serie de estrategias que consisten en la rehabilitación e intervención de los componentes del lenguaje (fonético, fonológico, morfosintáctico, semántico y pragmático) a través de ejercicios y actividades (estrategias) teniendo en cuenta los aporte teóricos de Víctor Acosta, Ana Moreno y Ángeles Axpe; los cuales hacen referencia a tres contextos importantes para la intervención en estos usuarios los cuales son el contexto académico, social y familiar (33),(34,)(35),(36).

Dicho diseño de estrategias se secciona por componentes lingüísticos y estos a su vez cuentan con su propio proceso de realización y objetivo, teniendo en cuenta el modelo de naturaleza dinámica de Víctor Acosta, donde habla que trabaja de manera transdisciplinar, donde se ha combinado el uso de procedimientos, estrategias, actividades y materiales, y aumentado la frecuencia e intensidad de las sesiones (37),(38), (39),(40).

Estas estrategias creadas se didactizaran en la creación de la página web "Creando Aprendizajes", donde las personas que ingresen podrán tener acceso a estas actividades de intervención para usuarios con TEL, esta página es un sistema aumentativo donde son utilizadas las Tecnologías de Información y Comunicación (TIC).

A continuación, en la Tabla 4 se evidencia la metodología GRADE que brinda resultados estadísticos para mayor precisión y así poder conocer cuál es el método más utilizado para la evaluación en infantes con Trastorno Específico del Lenguaje y qué estrategias terapéuticas a nivel de fonoaudiología se pueden implementar para niños con problemas en el desarrollo del lenguaje (3),(4),(5),(6),(7),(8),(9),(16),(17),(18)(19),(20),(21),(22),(23),(24),(41),(45),(46),(47).

TABLA 4. Metodología GRADE para la evaluación e intervención.

\begin{tabular}{|c|c|c|c|c|c|c|c|c|c|c|c|c|}
\hline \multicolumn{7}{|c|}{ Certainty assessment } & \multicolumn{2}{|c|}{ № de pacientes } & \multicolumn{2}{|c|}{ Efecto } & \multirow[b]{2}{*}{ Certainty } & \multirow[b]{2}{*}{$\begin{array}{c}\text { Importanc } \\
\text { ia }\end{array}$} \\
\hline $\begin{array}{c}\text { № de } \\
\text { estud } \\
\text { ios }\end{array}$ & $\begin{array}{c}\text { Diseño de } \\
\text { estudio }\end{array}$ & $\begin{array}{c}\text { Ries } \\
\text { go } \\
\text { de } \\
\text { sesg } \\
\text { o }\end{array}$ & $\begin{array}{c}\text { Inconsiste } \\
\text { ncia }\end{array}$ & $\begin{array}{c}\text { Eviden } \\
\text { cia } \\
\text { indirec } \\
\text { ta }\end{array}$ & $\begin{array}{l}\text { Impreci } \\
\text { sión }\end{array}$ & $\begin{array}{c}\text { Otras } \\
\text { consideraci } \\
\text { ones }\end{array}$ & $\begin{array}{l}\text { Método } \\
\text { de } \\
\text { rehabilita } \\
\text { ción en } \\
\text { pragmáti } \\
\text { ca }\end{array}$ & $\begin{array}{c}\text { mejor } \\
\text { ar los } \\
\text { proces } \\
\text { os } \\
\text { locutiv } \\
\text { os e } \\
\text { ilocuti } \\
\text { vos }\end{array}$ & $\begin{array}{l}\text { Relati } \\
\text { vo } \\
(95 \% \\
\text { CI) }\end{array}$ & $\begin{array}{c}\text { Absol } \\
\text { uto } \\
(95 \% \\
\text { CI) }\end{array}$ & & \\
\hline $\begin{array}{l}\text { Eval } \\
\text { prete }\end{array}$ & $\begin{array}{l}\text { e inter } \\
\text { est ) }\end{array}$ & & & & & & & & & & & aluación \\
\hline
\end{tabular}




\begin{tabular}{|c|c|c|c|c|c|c|c|c|c|c|c|c|}
\hline \multicolumn{7}{|c|}{ Certainty assessment } & \multicolumn{2}{|c|}{ № de pacientes } & \multicolumn{2}{|c|}{ Efecto } & \multirow[b]{2}{*}{ Certainty } & \multirow[b]{2}{*}{$\begin{array}{l}\text { Importanc } \\
\text { ia }\end{array}$} \\
\hline $\begin{array}{c}\text { № de } \\
\text { estud } \\
\text { ios }\end{array}$ & $\begin{array}{l}\text { Diseño de } \\
\text { estudio }\end{array}$ & $\begin{array}{c}\text { Ries } \\
\text { go } \\
\text { de } \\
\text { sesg } \\
\text { o }\end{array}$ & $\begin{array}{l}\text { Inconsiste } \\
\text { ncia }\end{array}$ & $\begin{array}{c}\text { Eviden } \\
\text { cia } \\
\text { indirec } \\
\text { ta }\end{array}$ & $\begin{array}{l}\text { Impreci } \\
\text { sión }\end{array}$ & $\begin{array}{c}\text { Otras } \\
\text { consideraci } \\
\text { ones }\end{array}$ & $\begin{array}{l}\text { Método } \\
\text { de } \\
\text { rehabilita } \\
\text { ción en } \\
\text { pragmáti } \\
\text { ca }\end{array}$ & $\begin{array}{c}\text { mejor } \\
\text { ar los } \\
\text { proces } \\
\text { os } \\
\text { locutiv } \\
\text { os e } \\
\text { ilocuti } \\
\text { vos }\end{array}$ & $\begin{array}{l}\text { Relati } \\
\text { vo } \\
(95 \% \\
\text { CI) }\end{array}$ & $\begin{array}{c}\text { Absol } \\
\text { uto } \\
(95 \% \\
\text { CI })\end{array}$ & & \\
\hline 1 & $\begin{array}{l}\text { estudios } \\
\text { observacio } \\
\text { nales }\end{array}$ & $\begin{array}{l}\text { no } \\
\text { es } \\
\text { serio }\end{array}$ & no es serio & $\begin{array}{l}\text { no es } \\
\text { serio }\end{array}$ & $\begin{array}{ll}\text { no } & \text { es } \\
\text { serio } & \end{array}$ & $\begin{array}{l}\text { fuerte } \\
\text { asociación }\end{array}$ & $\begin{array}{l}5 / 5 \\
(100.0 \%)\end{array}$ & $\begin{array}{l}5 / 5 \\
(100.0 \\
\%)\end{array}$ & $\begin{array}{l}\mathbf{R R} \\
\mathbf{0 . 5 5} \\
(0.30 \mathrm{a} \\
0.60)\end{array}$ & $\begin{array}{l}\mathbf{4 5} \\
\text { menos } \\
\text { por } \\
\mathbf{1 0 0} \\
\text { (de } 40 \\
\text { menos } \\
\text { a } 70 \\
\text { menos } \\
\text { ) }\end{array}$ & $\begin{array}{l}\oplus \oplus \oplus \bigcirc \\
\text { MODER } \\
\text { ADO }\end{array}$ & $\begin{array}{l}\text { IMPORTA } \\
\text { NTE }\end{array}$ \\
\hline \multicolumn{13}{|c|}{$\begin{array}{l}\text { Intervención en trastornos pragmáticos: consideraciones metodológicas (evaluado con : Revisión de los principales modelos interpretativos de los } \\
\text { trastornos pragmáticos ) }\end{array}$} \\
\hline 1 & $\begin{array}{l}\text { estudios } \\
\text { observacio } \\
\text { nales }\end{array}$ & $\begin{array}{l}\text { no } \\
\text { es } \\
\text { serio }\end{array}$ & no es serio & $\begin{array}{l}\text { no es } \\
\text { serio }\end{array}$ & $\begin{array}{l}\text { no es } \\
\text { serio }\end{array}$ & ninguno & & & $\begin{array}{c}\text { no } \\
\text { estima } \\
\text { ble }\end{array}$ & & $\begin{array}{l}\oplus \oplus \bigcirc \\
\text { BAJA }\end{array}$ & $\begin{array}{l}\text { NO ES } \\
\text { IMPORTA } \\
\text { NTE }\end{array}$ \\
\hline \multicolumn{13}{|c|}{ Efectividad de una intervención de comunicación social manualizada e intensiva (SCIP) para niños que tienen PLI con o sin características de ASD } \\
\hline & est & & & & & & \multicolumn{2}{|c|}{4 casos 0 Controles } & \multirow{2}{*}{$\begin{array}{c}\mathbf{R R} \\
\mathbf{0 . 3} \\
(0.3 \mathrm{a} \\
0.5)\end{array}$} & - & & \\
\hline 1 & $\begin{array}{l}\text { observacio } \\
\text { nales }\end{array}$ & $\begin{array}{l}\text { es } \\
\text { serio }\end{array}$ & no es serio & $\begin{array}{l}\text { no es } \\
\text { serio }\end{array}$ & $\begin{array}{l}\text { no es } \\
\text { serio }\end{array}$ & ninguno & - & $70.0 \%$ & & $\begin{array}{c}49 \\
\text { menos } \\
\text { por } 100 \\
\text { (de } 35 \\
\text { menos } \\
\text { a } 49 \\
\text { menos) }\end{array}$ & $\begin{array}{l}\oplus \oplus \bigcirc \\
\text { BAJA }\end{array}$ & $\begin{array}{l}\text { NO ES } \\
\text { IMPORTA } \\
\text { NTE }\end{array}$ \\
\hline \multicolumn{13}{|c|}{$\begin{array}{l}\text { Habilidades pragmáticas en niños con desvío fonológico (evaluado con : Análisis de los aspectos pragmáticos de estos niños, utilizando el } \\
\text { instrumento ABFW) }\end{array}$} \\
\hline \multirow[b]{2}{*}{1} & \multirow[b]{2}{*}{$\begin{array}{l}\text { estudios } \\
\text { observacio } \\
\text { nales }\end{array}$} & \multirow[b]{2}{*}{$\begin{array}{l}\text { no } \\
\text { es } \\
\text { serio }\end{array}$} & \multirow[b]{2}{*}{ no es serio } & \multirow[b]{2}{*}{$\begin{array}{l}\text { no es } \\
\text { serio }\end{array}$} & \multirow[b]{2}{*}{$\begin{array}{l}\text { no es } \\
\text { serio }\end{array}$} & \multirow[b]{2}{*}{$\begin{array}{l}\text { Fuerte } \\
\text { asociación }\end{array}$} & \multicolumn{2}{|l|}{$\begin{array}{l}12 \text { casos } 12 \\
\text { Controles }\end{array}$} & \multirow{2}{*}{$\begin{array}{c}\mathbf{R R} \\
\mathbf{0 . 9} \\
(0.8 \mathrm{a} \\
0.9)\end{array}$} & - & \multirow{2}{*}{$\begin{array}{c}\oplus \oplus \oplus \bigcirc \\
\text { MODER } \\
\text { ADO }\end{array}$} & \multirow[t]{2}{*}{$\begin{array}{l}\text { IMPORTA } \\
\text { NTE }\end{array}$} \\
\hline & & & & & & & - & $10.0 \%$ & & $\begin{array}{c}\mathbf{1} \\
\text { menos } \\
\text { por } \\
\mathbf{1 0 0} \\
\text { (de } 1 \\
\text { menos } \\
\text { a } 2 \\
\text { menos } \\
\text { ) }\end{array}$ & & \\
\hline
\end{tabular}

Fuente: Autores.

Habilidades pragmáticas en niños con desvío fonológico (evaluado con : Análisis de los aspectos pragmáticos de estos niños, utilizando el instrumento ABFW)

\begin{tabular}{|l|l|l|l|l|l|l|l|l|l|l|l|}
\hline & & & & & & & $\begin{array}{l}12 \text { casos 12 } \\
\text { Controles }\end{array}$ & $\begin{array}{l}\text { RR } \\
0.9\end{array}$ & - & $\oplus \oplus \oplus \bigcirc$ & IMPORTANTE \\
\hline
\end{tabular}




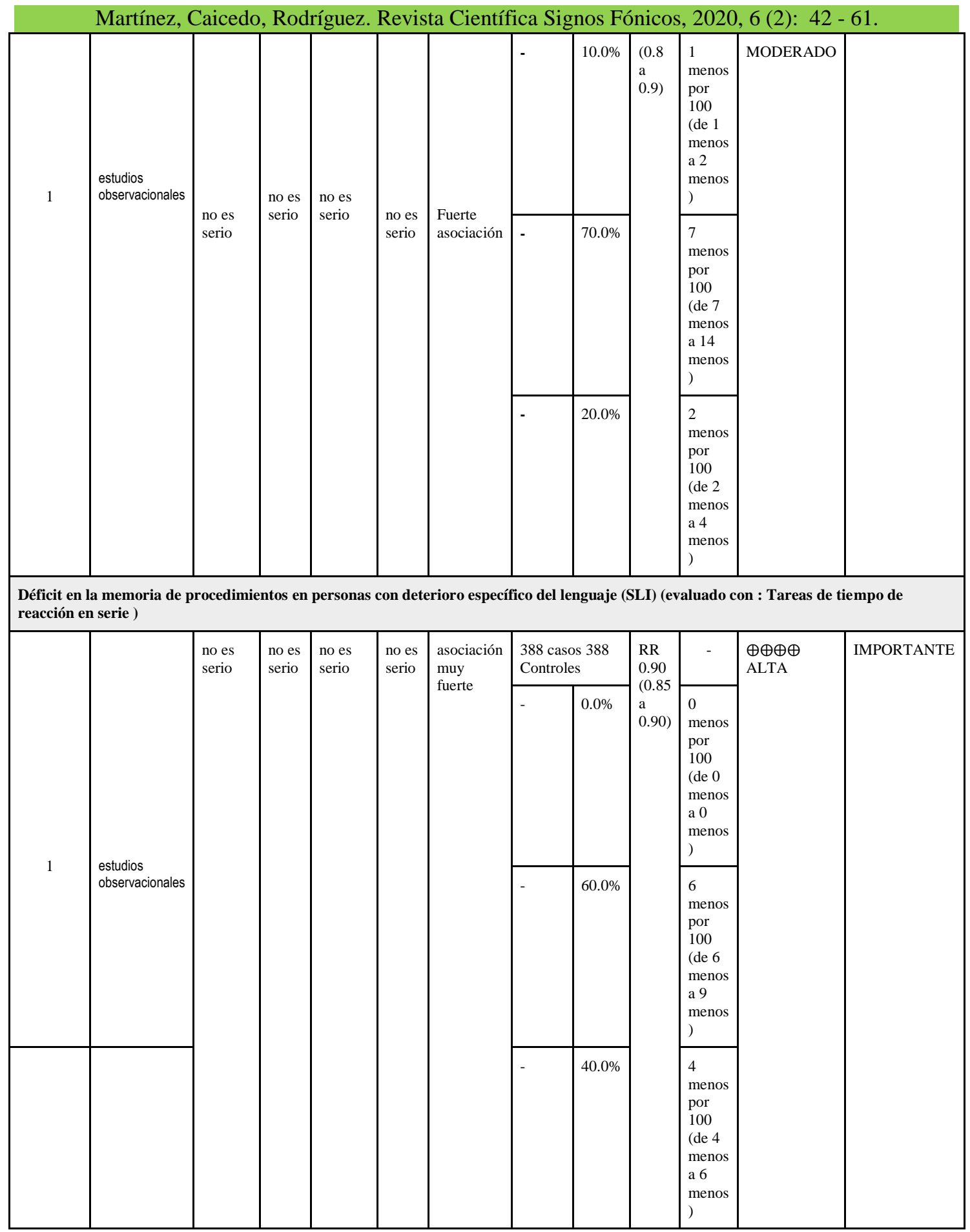

TABLA 4. Metodología GRADE para la evaluación e intervención.

Fuente: Autores.

TABLA 4. Metodología GRADE para la evaluación e intervención. 


\begin{tabular}{|c|c|c|c|c|c|c|c|c|c|c|c|c|}
\hline \multicolumn{13}{|c|}{ Martínez, Caicedo, Rodríguez. Revista Científica Signos Fónicos, 2020, 6 (2): 42 - 61.} \\
\hline 1 & \begin{tabular}{|l} 
ensayos \\
aleatorios
\end{tabular} & $\begin{array}{l}\text { no es } \\
\text { serio }\end{array}$ & no es serio & $\begin{array}{l}\text { no es } \\
\text { serio }\end{array}$ & $\begin{array}{l}\text { no es } \\
\text { serio }\end{array}$ & ninguno & $\begin{array}{c}16 / 16 \\
(100.0 \%)\end{array}$ & & $\begin{array}{c}\text { RR } 0.50 \\
(0.30 \mathrm{a} \\
0.55)\end{array}$ & $\begin{array}{c}1 \\
\text { menos } \\
\text { por } \\
100 \\
\text { (de } 0 \\
\text { menos } \\
\text { a } 1 \\
\text { menos } \\
\text { ) }\end{array}$ & $\underset{\text { ALTA }}{\bigoplus \bigoplus \bigoplus \bigoplus}$ & IMPORTANTE \\
\hline \multicolumn{13}{|c|}{ Trastorno Específico del Desarrollo del Lenguaje en una población infantil colombiana (evaluado con : Evaluación neuropsicológica y lingüística ) } \\
\hline 1 & \begin{tabular}{|l|} 
estudios \\
observacionales
\end{tabular} & $\begin{array}{l}\text { no es } \\
\text { serio }\end{array}$ & no es serio & $\begin{array}{l}\text { no es } \\
\text { serio }\end{array}$ & $\begin{array}{l}\text { no es } \\
\text { serio }\end{array}$ & $\begin{array}{l}\text { asociación } \\
\text { muy fuerte }\end{array}$ & $\begin{array}{c}204 / 51 \\
(400.0 \%)\end{array}$ & - & - & - & $\underset{\text { ALTA }}{\bigoplus \bigoplus \bigoplus \bigoplus}$ & IMPORTANTE \\
\hline \multicolumn{13}{|c|}{ Trastorno Específico del Lenguaje: Marcadores psicolingüisticos en semántica y pragmática en niños españoles (evaluado con : Tareas psicolingüisticas) } \\
\hline \multirow[t]{3}{*}{1} & \multirow[t]{3}{*}{$\begin{array}{l}\text { estudios } \\
\text { observacionales }\end{array}$} & \multirow[t]{3}{*}{$\begin{array}{l}\text { no es } \\
\text { serio }\end{array}$} & \multirow[t]{3}{*}{ no es serio } & \multirow[t]{3}{*}{$\begin{array}{l}\text { no es } \\
\text { serio }\end{array}$} & \multirow[t]{3}{*}{$\begin{array}{l}\text { no es } \\
\text { serio }\end{array}$} & \multirow[t]{3}{*}{$\begin{array}{l}\text { asociación } \\
\text { muy fuerte }\end{array}$} & \multicolumn{2}{|c|}{$\begin{array}{l}31 \text { casos } 31 \text { Controles } \\
31 / 31 \text { expuesto } 0 / 0 \text { no } \\
\text { expuesto }\end{array}$} & \multirow[t]{3}{*}{$\begin{array}{c}\text { RR } 0.7 \\
(0.6 \mathrm{a} \\
0.8)\end{array}$} & - & \multirow[t]{3}{*}{$\underset{\text { ALTA }}{\bigoplus \bigoplus \bigoplus \bigoplus}$} & \multirow[t]{3}{*}{ IMPORTANTE } \\
\hline & & & & & & & - & $10.0 \%$ & & $\begin{array}{c}3 \\
\text { menos } \\
\text { por } \\
100 \\
\text { (de } 2 \\
\text { menos } \\
\text { a } 4 \\
\text { menos } \\
\text { ) }\end{array}$ & & \\
\hline & & & & & & & - & $90.0 \%$ & & $\begin{array}{c}27 \\
\text { menos } \\
\text { por } \\
100 \\
\text { (de } 18 \\
\text { menos } \\
\text { a } 36 \\
\text { menos } \\
\text { ) }\end{array}$ & & \\
\hline \multicolumn{13}{|c|}{ Programa de intervención en lectura temprana en Educación In-fantil en seis escolares con trastorno específico del lenguaje (evaluado con : Evolución de los alumnos) } \\
\hline 1 & \begin{tabular}{|l|} 
estudios \\
observacionales
\end{tabular} & $\begin{array}{l}\text { no es } \\
\text { serio }\end{array}$ & no es serio & $\begin{array}{l}\text { no es } \\
\text { serio }\end{array}$ & $\begin{array}{l}\text { no es } \\
\text { serio }\end{array}$ & ninguno & $\begin{array}{c}17 / 17 \\
(100.0 \%)\end{array}$ & - & - & - & $\underset{\text { BAJA }}{\oplus \oplus \bigcirc}$ & $\begin{array}{l}\text { NO ES } \\
\text { IMPORTANTE }\end{array}$ \\
\hline
\end{tabular}

CI: Intervalo de confianza. RR: Razón de riesgo.

Fuente: Autores.

En la Tabla 5 se presentan también los mismos resultados en una tabulación con metodología GRADE ya que es el método que nos arroga datos estadísticos y nos da mayor precisión al momento de determinar la respuesta y la conclusión, donde nos muestra la validez de cada base teórica y la fuerza que representa la evidencia científica en esta pesquisa (3), (4), (5), (6), (7), (8), (9), (16), (17), (18), (19), (20), (21), (22), (23), (24), (41), (45), (46), (47). 
Tabla 5. Resultados estadísticos con metodología GRADE para la evaluación intervención.

Método de evaluación e intervención comparado para mejorar los procesos comunicativos en usuarios con TEL

Paciente o población: usuarios con TEL

Configuración: Intervención: Método de rehabilitación en pragmática. Comparación: mejorar los procesos locutivos e ilocutivos

\begin{tabular}{|c|c|c|c|c|c|c|}
\hline \multirow[t]{2}{*}{ Desenlaces } & \multicolumn{2}{|c|}{$\begin{array}{l}\text { Efectos absolutos } \\
\text { anticipados * (95\% CI) }\end{array}$} & \multirow{2}{*}{$\begin{array}{l}\text { Efecto } \\
\text { relativo } \\
(95 \% \mathrm{CI})\end{array}$} & \multirow{2}{*}{$\begin{array}{l}\text { № de } \\
\text { participantes } \\
\text { (Estudios) }\end{array}$} & \multirow{2}{*}{$\begin{array}{l}\text { Certainty of } \\
\text { the evidence } \\
\text { (GRADE) }\end{array}$} & \multirow[t]{2}{*}{ Comentarios } \\
\hline & $\begin{array}{l}\text { Riesgo } \\
\text { con } \\
\text { mejorar } \\
\text { los } \\
\text { procesos } \\
\text { locutivos e } \\
\text { ilocutivos }\end{array}$ & $\begin{array}{l}\text { Riesgo con } \\
\text { Método de } \\
\text { rehabilitación } \\
\text { en } \\
\text { pragmática }\end{array}$ & & & & \\
\hline $\begin{array}{l}\text { Evaluación e } \\
\text { intervención } \\
\text { pragmática en } \\
\text { niños con } \\
\text { medidas de } \\
\text { protección } \\
\text { evaluado con: } \\
\text { Evaluación } \\
\text { pretest-postest } \\
\text { seguimiento: } \\
\text { rango } 1 \text { semanas } \\
\text { a } 4 \text { semanas }\end{array}$ & $\begin{array}{l}100 \text { por } \\
100\end{array}$ & $\begin{array}{l}55 \text { por } 100 \\
(30 \text { a } 60)\end{array}$ & $\begin{array}{l}\text { RR 0.55 } \\
(0.30 \mathrm{a} \\
0.60)\end{array}$ & $\begin{array}{l}10 \\
\text { (1 estudio } \\
\text { observacional) }\end{array}$ & $\begin{array}{l}\bigoplus \bigoplus \oplus \bigcirc \\
\text { MODERADO }\end{array}$ & \\
\hline $\begin{array}{l}\text { Intervención en } \\
\text { trastornos } \\
\text { pragmáticos: } \\
\text { consideraciones } \\
\text { metodológicas } \\
\text { evaluado con: } \\
\text { Revisión de los } \\
\text { principales } \\
\text { modelos } \\
\text { interpretativos de } \\
\text { los trastornos } \\
\text { pragmáticos }\end{array}$ & 0 por 100 & $\begin{array}{l}\text { 0 por } 100 \\
(0 \text { a } 0)\end{array}$ & $\begin{array}{l}\text { no } \\
\text { estimable }\end{array}$ & $\begin{array}{l}\text { (1 estudio } \\
\text { observacional) }\end{array}$ & $\underset{\text { BAJA }}{\oplus \oplus \bigcirc}$ & \\
\hline Efectividad de & Bajo & & RR 0.3 & 4 casos 0 & $\oplus \oplus \bigcirc$ & \\
\hline
\end{tabular}


Método de evaluación e intervención comparado para mejorar los procesos comunicativos en usuarios con TEL

Paciente o población: usuarios con TEL

Configuración: Intervención: Método de rehabilitación en pragmática. Comparación: mejorar los procesos locutivos e ilocutivos

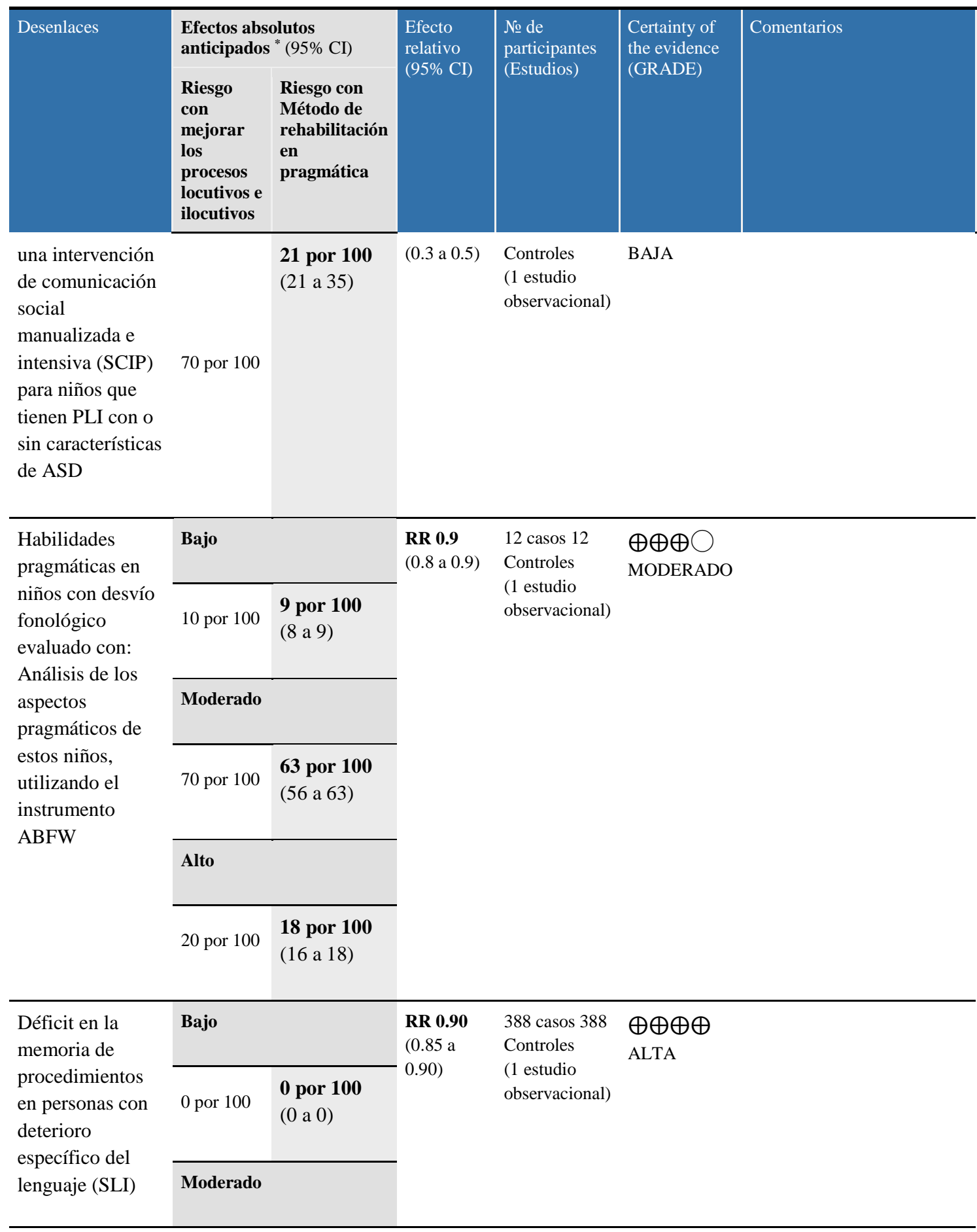


Método de evaluación e intervención comparado para mejorar los procesos comunicativos en usuarios con TEL

Paciente o población: usuarios con TEL

Configuración: Intervención: Método de rehabilitación en pragmática. Comparación: mejorar los procesos locutivos e ilocutivos

\begin{tabular}{|c|c|c|c|c|c|c|}
\hline \multirow[t]{2}{*}{ Desenlaces } & \multicolumn{2}{|c|}{$\begin{array}{l}\text { Efectos absolutos } \\
\text { anticipados * }(95 \% \mathrm{CI})\end{array}$} & \multirow{2}{*}{$\begin{array}{l}\text { Efecto } \\
\text { relativo } \\
\text { (95\% CI) }\end{array}$} & \multirow{2}{*}{$\begin{array}{l}\text { № de } \\
\text { participantes } \\
\text { (Estudios) }\end{array}$} & \multirow{2}{*}{$\begin{array}{l}\text { Certainty of } \\
\text { the evidence } \\
\text { (GRADE) }\end{array}$} & \multirow[t]{2}{*}{ Comentarios } \\
\hline & $\begin{array}{l}\text { Riesgo } \\
\text { con } \\
\text { mejorar } \\
\text { los } \\
\text { procesos } \\
\text { locutivos e } \\
\text { ilocutivos }\end{array}$ & $\begin{array}{l}\text { Riesgo con } \\
\text { Método de } \\
\text { rehabilitación } \\
\text { en } \\
\text { pragmática }\end{array}$ & & & & \\
\hline \multirow{3}{*}{$\begin{array}{l}\text { evaluado con: } \\
\text { Tareas de tiempo } \\
\text { de reacción en } \\
\text { serie }\end{array}$} & 60 por 100 & $\begin{array}{l}54 \text { por } 100 \\
(51 \text { a } 54)\end{array}$ & & & & \\
\hline & \multicolumn{2}{|l|}{ Alto } & & & & \\
\hline & 40 por 100 & $\begin{array}{l}\mathbf{3 6} \text { por } 100 \\
(34 \text { a } 36)\end{array}$ & & & & \\
\hline $\begin{array}{l}\text { Diseño de un } \\
\text { método rápido de } \\
\text { evaluación que } \\
\text { proporcionará } \\
\text { una evaluación } \\
\text { general rápida de } \\
\text { la efectividad } \\
\text { pragmática de los } \\
\text { hablantes } \\
\text { afectados } \\
\text { neurológicamente } \\
\text { evaluado con: } \\
\text { Evaluación } \\
\text { pragmática }\end{array}$ & 0 por 100 & $\begin{array}{l}\text { 0 por } 100 \\
(0 \text { a } 0)\end{array}$ & $\begin{array}{l}\text { RR 0.50 } \\
(0.30 \mathrm{a} \\
0.55)\end{array}$ & $\begin{array}{l}16 \\
(1 \text { ECA } \\
\text { (experimento } \\
\text { controlado } \\
\text { aleatorizado)) }\end{array}$ & $\begin{array}{l}\bigoplus \bigoplus \bigoplus \bigoplus \\
\text { ALTA }\end{array}$ & \\
\hline $\begin{array}{l}\text { Trastorno } \\
\text { Específico del } \\
\text { Desarrollo del } \\
\text { Lenguaje en una } \\
\text { población infantil } \\
\text { colombiana } \\
\text { evaluado con: } \\
\text { Evaluación } \\
\text { neuropsicológica } \\
\text { y lingüística }\end{array}$ & - & - & - & $\begin{array}{l}51 \\
\text { (1 estudio } \\
\text { observacional) }\end{array}$ & $\begin{array}{l}\bigoplus \oplus \bigoplus \bigoplus \\
\text { ALTA }\end{array}$ & \\
\hline
\end{tabular}


Método de evaluación e intervención comparado para mejorar los procesos comunicativos en usuarios con TEL

Paciente o población: usuarios con TEL

Configuración: Intervención: Método de rehabilitación en pragmática. Comparación: mejorar los procesos locutivos e ilocutivos

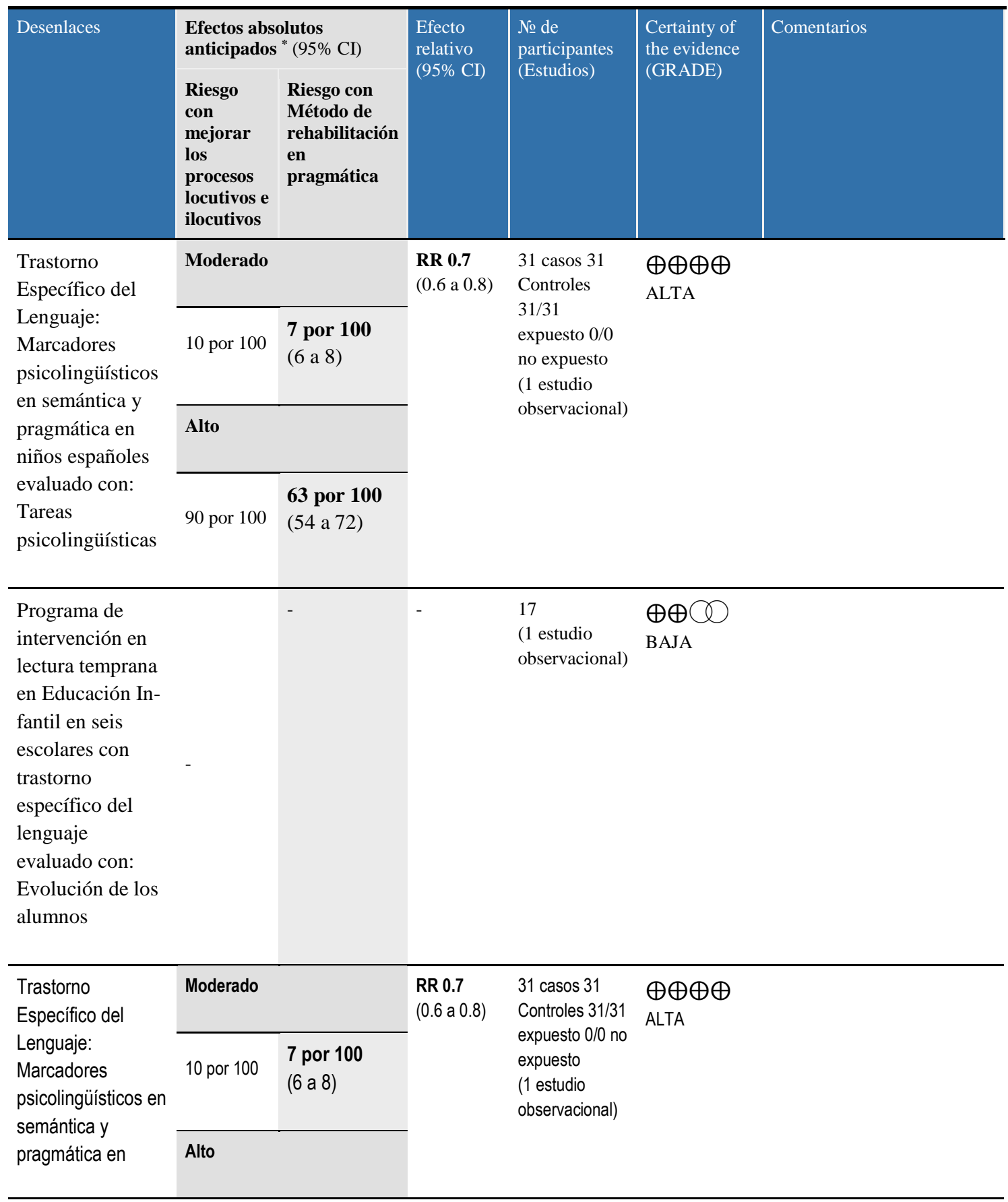


Método de evaluación e intervención comparado para mejorar los procesos comunicativos en usuarios con TEL

Paciente o población: usuarios con TEL

Configuración: Intervención: Método de rehabilitación en pragmática. Comparación: mejorar los procesos locutivos e ilocutivos

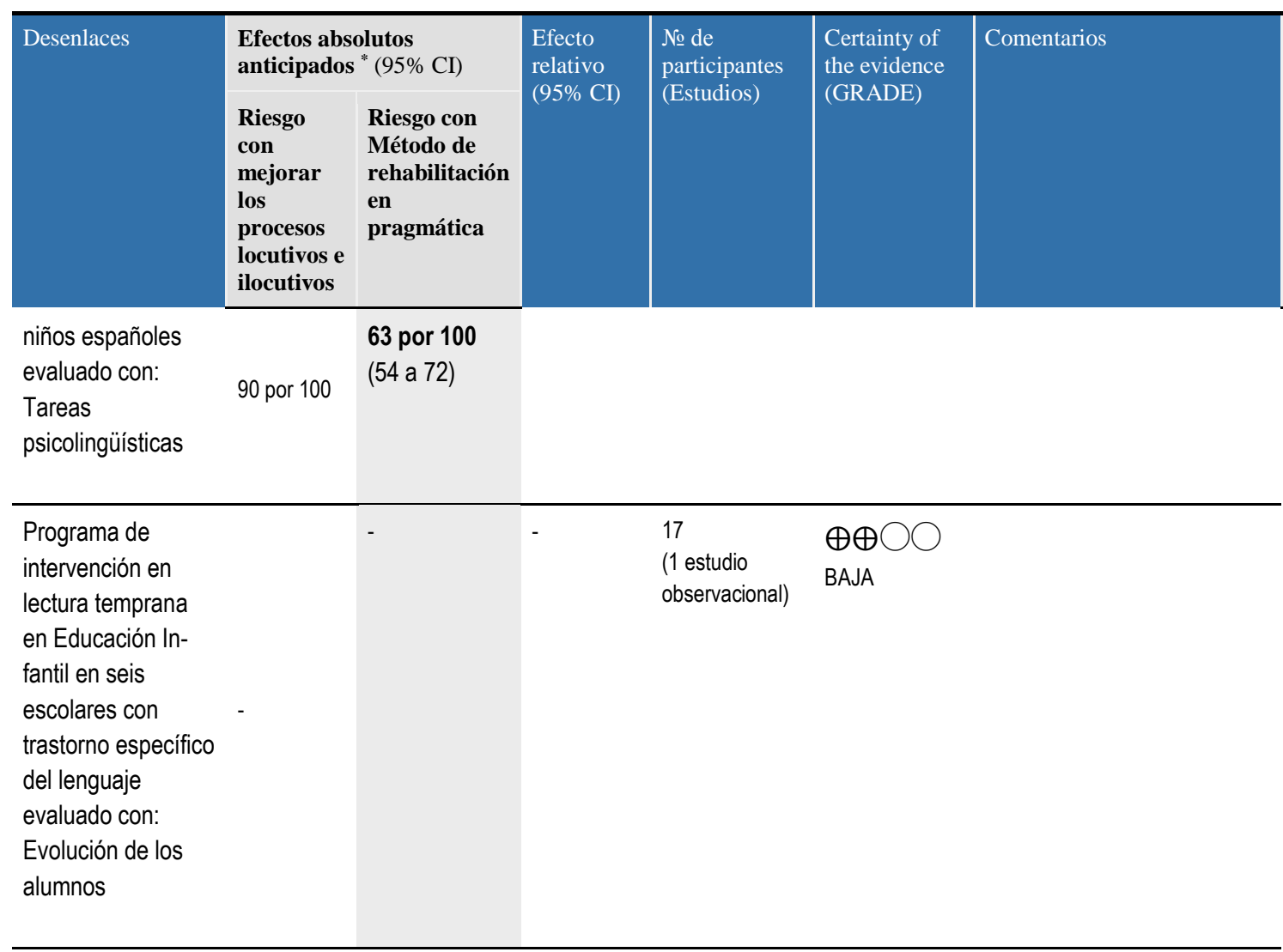

El riesgo en el grupo de intervención (y su intervalo de confianza del 95\%) se basa en el riesgo asumido en el grupo de comparación y en el efecto relativo de la intervención (y su intervalo de confianza del 95\%).

CI: Intervalo de confianza; RR: Razón de riesgo

\section{GRADE Working Group grades of evidence}

High certainty: We are very confident that the true effect lies close to that of the estimate of the effect

Moderate certainty: We are moderately confident in the effect estimate: The true effect is likely to be close to the estimate of the effect, but there is a possibility that it is substantially different

Low certainty: Our confidence in the effect estimate is limited: The true effect may be substantially different from the estimate of the effect

Very low certainty: We have very little confidence in the effect estimate: The true effect is likely to be substantially different from the estimate of effect

Fuente: Autores. 


\begin{abstract}
ANÁLISIS Y DISCUSIÓN
Dentro de la revisión bibliográfica el método buscado consta de la evaluación e intervención en infantes con TEL. Se destaca en esta pesquisa que un método utilizado para la anterior finalidad mencionada es el método de los Modelos de Adams, con aportes de Freeman y Dake, Schelstraete con bases en el modelo MEHRIT (4),(42),(43),(44); donde nos menciona que dicha evaluación se debe seccionar en tres modelos principales: el social, que nos menciona la relación y la inferencia social, el cognitivo, el cual nos enfatiza el déficit de cognición social; es decir la capacidad de captar los mensajes propuestos por el entorno, y el lingüístico que nos dirige a las consecuencias de fallo en las competencias de observación, imitación y procesos de ensayo-error.
\end{abstract}

También se resaltan los aportes de Freeman y Dake, en donde se expresan los modelos de Adams simplificados en dos, como especie de adaptación entre los que se encuentran el social y el naturalístico (5),(45),(46),(47); para que el resultado del método sea efectivo, se evidencia que el protocolo cuenta con nuevos aportes o adaptaciones, que también nos indican que los modelos de Adams son adecuados y que se pueden adaptar según las necesidades del usuario.

Dentro de las secciones del protocolo se observa la importancia de aspectos sociales, cognitivos y linguísticos, para la mejoría de los infantes a intervenir. Igualmente resalta dentro del mismo análisis que dicha metodología presenta la línea de fuerza principal en este estudio el cual es el Trastorno Específico del Lenguaje, que se considera una condición que retrasa la adquisición del lenguaje, que se correlaciona con los trastornos específicos del aprendizaje, puesto que se requiere de los procesos de aprendizaje, para la adquisición de las habilidades lingüísticas (48),(49),(50).

Teniendo en cuenta como fuente principal los artículos de esta investigación que son de carácter preciso y conciso, la cantidad de referentes teóricos encontrados fueron limitados, pues el campo que se abordó no es muy usual en investigaciones; por lo mismo que se acaba de mencionar se considera necesario la realización de nuevos métodos de intervención presentando los limitantes necesarios como el Trastorno Especifico del Lenguaje. A partir de este resultado se realiza el diseño de estrategias para la intervención teniendo en cuenta La intervención en niños con TEL debe plantearse desde un punto de vista multidisciplinar (lingüístico, psicológico, escolar, familiar, etc.), ya que Limitarse únicamente al tratamiento del lenguaje, sería cerrar muchas posibilidades al desarrollo integral del niño (51), (52), (53). Cabe resaltar que Víctor Acosta nos sugiere que al momento de la intervención con estos usuarios se debe tener en cuenta los contextos académicos, sociales y familiares tanto para la evaluación como la intervención (54), (55), (56).

\title{
CONCLUSIONES
}

- Hay que tener en cuenta las enormes dificultades para encontrar sujetos con un Trastorno Específico del Lenguaje, especialmente con un diagnóstico certero a edades tan tempranas.

- Se afirma que existen diversos métodos de evaluación para usuarios con TEL que tienen como objetivo evaluar procesos como atención, concentración, memoria de trabajo, producción de 
Martínez, Caicedo, Rodríguez. Revista Científica Signos Fónicos, 2020, 6 (2): 42 - 61.

fonemas vocálicos y consonánticos, discriminación auditiva, segmentación silábica, repetición de palabras y pseudopalabras, y cohesión coherencia en el discurso.

- Se encontró una relación entre TEA (trastorno especifico del aprendizaje) y TEL (trastorno especifico del lenguaje) ya que si un usuario presenta un trastorno de aprendizaje no podrá integrar el lenguaje.

- Tanto para la evaluación como la intervención se debe llevar a cabo los procesos mediante diferentes contextos donde se desarrolla el infante.

- Estas estrategias pertenecientes a "Creando Aprendizajes" permiten favorecer las habilidades comunicativas para un aprendizaje orientada a usuarios con un Trastorno Específico del Lenguaje de una forma dinámica desarrollando destrezas auditivas, visuales, cognitivas y lingüísticas.

- Creando Aprendizajes son estrategias terapéuticas encaminadas a la intervención de infantes con TEL en edades comprendidas desde los 3 a los 7 años de edad desde el abordaje de cada uno de los componentes del lenguaje.

- Se requiere continuar con la retroalimentación de la página web "Creando Aprendizajes" para así generar nuevas fases en el proceso.

\section{REFERENCIAS BIBLIOGRÁFICAS}

1.NIH. NIDCD (MD): U.S. National intitute of health. Dental health. (actualizado 2009; el accesso en el año 2020] Disponible en: https://www.nidcd.nih.gov.html

2.Mogollón M, Portilla E. Metodologías de Evaluación Fonoaudiológica del Componente Pragmático del Lenguaje en Infantes. Revista Signos Fónicos. 2015;1(1):1-10.Disponible en: http://revistas.unipamplona.edu.co/ojs_viceinves/index.php/CDH/article/view/1320/843

3. López-Contreras, Luísa-Fernanda. Grado De Severidad De Disfonía A Través Del Análisis Acústico Vocal En Docentes De Dos Instituciones De La Estrategia De Atención A La Primera Infancia. Revistas Científica Signos Fónicos. 2019: 5 (1). 1-9, https://doi.org/10.24054/01204211.v1.n1.2019.3976.

4.Monfort M, Sánchez A. Intervención en trastornos pragmáticos: consideraciones metodológicas. Artículo Elsevier. 2018, 38 (1): 24-30. Disponible en: https://www.elsevier.es/es-revista-revistalogopedia-foniatria-audiologia-309-articulo-intervencion-trastornos-pragmaticos-consideracionesmetodologicas-S0214460317300955

5.Adams, C, Lockton, E, Freed, J., Gaile, J., Earl, G., McBean, K., Law, J.The Social Communication Intervention Project: a randomized controlled trial of the effectiveness of speech and language therapy for school-age children who have pragmatic and social communication problems with or without autism spectrum disorder. International Journal of Language \& Communication Disorders. 2010; 47(3): 233244. Doi.Org/10.1111/J.1460-6984.2011.00146.X

6.Haverkamp, F, Mohamad, Y. Need and Perspectives of Internet-based Interventions for Common Specific language Disorders and Connected Specific Learning Disabilities in Childhood and Youth. Procedia Computer Science. 2015, 67, 439-444. Disponible en: https://doi.org/10.1016/j.procs.2015.11.084

7.Hincapié, L. Giraldo, M. Lopera, F. Pineda, D. Castro, R. Lopera, J. Trastorno específico del desarrollo del lenguaje en una población infantil colombiana. UNIV. PSYCHOL. 2008, 7 (2): 557-569. 
8.Buiza, J. J., Rodríguez-Parra, M. J., \& Adrián, J. A. (2015). Trastorno Específico del Lenguaje: Marcadores psicolingüísticos en semántica y pragmática en niños españoles. Anales de Psicologia, 31(3), 879-889. Disponible en: https://doi.org/10.6018/analesps.31.3.180091

9.Rodríguez A., Droguett L, Revuelta, L. Ajuste escolar y personal en la adolescencia: El papel del autoconcepto academic y del apoyo social percibido. Revista de Psicodidactica. 2012, 17(2), $397-414$. Doi.Org/10.1387/Rev.Psicodidact.4496

10.Narbona J. Subtipos de trastorno específico del desarrollo del lenguaje: perfiles clínicos en una muestra hispanohablante. 2006;43(1):24-30.

11. Cáceres-Mansilla, Luz-Amparo. Sindrome de Burnout, Trastornos del Sueño y su asociación con las alteraciones vocales en docentes de primera infancia. Revistas Científica Signos Fónicos. 2019 : 5 (1). 10-19, https://doi.org/10.24054/01204211.v1.n1.2019.3977

12. Guerrero M L, Caicedo T. Method of rehabilitation in pragmatics to improve the local and successful process in users with specific disorder of language. Systematic review. 2018.

13.Manterola C, Astudillo P, Arias E, Claros N. Revisiones sistemáticas de la literatura. Qué se debe saber acerca de ellas. Elsevier. 2013; 3: 149-155.

14.Landa E, Arredondo A. Herramienta PICO para la formulación y búsqueda de preguntas clínicamente relevantes en la psicooncología basada en la evidencia [Internet]. 2014;11(3):259-270. DOI: $10.5209 /$

15. Navarro-Criollo; Paola-Lorena. Validación de indicadores de perturbación de Jitter y Shimmer en docentes de primera infancia. Revistas Científica Signos Fónicos. 2019: 5 (1). 20-29, https://doi.org/10.24054/01204211.v1.n1.2019.3978

16.Zens N K, Gillon G T, Moran C. Effects of phonological awareness and semantic intervention on word-learning in children with SLI. International Journal of Speech-Language Pathology, 11(6), 509524. Doi.Org/10.3109/17549500902926881

17.Vydrova R, Komarek V, Sanda J, Sterbova K, Jahodova A, Maulisova A, Kyncl M.Structural alterations of the language connectome in children with specific language impairment. Brain and Language.2015; 151: 35-41. Doi.Org/10.1016/J.Band1.2015.10.003

18. Acosta V, Ramírez G, Hernández S.Executive functions and language in children with different subtypes of specific language impairment. Neurología.2017; 32(6):355-362. doi.org/10.1016/j.nrleng.2015.12.007

19.Barini NS, Hage S R D V. Vocabulário e compreensão verbal de escolares com Transtorno do Déficit de Atenção e Hiperatividade. CoDAS.2015;27(5):446-451.Doi.Org/10.1590/2317$1782 / 20152015022$

20. Acosya V, Moreno A. M, Axpe M. Intervención sobre la conciencia fonológica en sujetos con trastorno específico del lenguaje en contextos inclusivos: posibilidades y limitaciones. Bordón. Revista De Pedagogía. 2011; 63 (3): 9-22.

21. Dybkjaer L, Bernsen NO, Dybkjaer H, McKelvie D, Mengel A. The MATE Markup Framework. MATE Deliv.1998. 
Martínez, Caicedo, Rodríguez. Revista Científica Signos Fónicos, 2020, 6 (2): 42 - 61.

22. Adams C. Clinical diagnostic and intervention studies of children with semantic- pragmatic language disorder. International Journal 01 Language and Communication Disorders. 2001; 36: 289305. DOI: $10.1080 / 13682820119881$

23.Adams C, Bisnop O. Conversational characteristics of children with semantic- prag- mane disorders. 1: Exchange structure. turn-taking. repairs and conesíon. British Journal ol Oisorders 01Communication. 1989;24 (3): 211-240. DOI: 10.3109/13682828909019889

24.Adams, C. Clarke, E. y Haynes. R. Inference and sentence comprehension in children with specific or pragmatic language impairments. International Journal 01 Language and Communication Disorders. 2009; 44 (3): 301- 318. DOI: 10.1080/13682820802051788

25. Carvajal-Villamizar,Yuliana-Mercedes. Actitudes de los docentes y estudiantes hacia las personas con discapacidad en una institución pública. Revistas Científica Signos Fónicos. 2019: 5 (1). 30-44, https://doi.org/10.24054/01204211.v1.n1.2019.3979

26. Acosta V, Moreno A, Axpe Á. Implicaciones clínicas del diagnóstico diferencial temprano entre Retraso de Lenguaje (RL) y Trastorno Específico del Lenguaje (TEL). Universitas Psychologica [Internet]. 2012;11(1):279-291.

27.Moreno Santana, A, Axpe Caballero, Á, Acosta Rodríguez, V. La acción inclusiva para la mejora de habilidades de lenguaje oral y de lectura inicial en niños con Trastorno Específico del Lenguaje (TEL). Universidad de la Lagua. 2012; 359: 322-356.

28.Del Valle N, De Castro L, Acosta D. La evaluación e intervención narrativa en niños con TEL. Logopedia: Evaluación, transformación y futuro. 2014; 246-256.

29.Roqueta C, Clemente E, Flores R. Cognición Social y competencia pragmática. El caso de los niños y niñas con Trastorno Específico del Lenguaje. International Journal of Psychological Research [Internet]. 2012;5(1):59-69.

30.Moreno A, Axpe Á, Acosta V. Efectos de un programa de intervención en el lenguaje sobre el desarrollo del léxico y del procesamiento fonológico en escolares de educación infantil con trastorno específico del lenguaje. Revista de Investigación Educativa. 2012;30(1):71-86.

31. Martínez-Siza, Diana-Carolina. Creencias de los docentes acerca de la educación inclusiva: un estudio fenomenológico. Revistas Científica Signos Fónicos. 2019: 5 (1). 45-59, https://doi.org/10.24054/01204211.v1.n1.2019.3980

32.Acosta A, Moreno A. Guía de actuaciones educativas en el ámbito de la comunicación y el lenguaje. Consejería de Educación, Universidades, Cultura y Deportes del gobierno de Canarias. Dirección General de Ordenación e Innovación Educativa. 2007;6-60.

33.Acosta V, Ramírez G, Hernández S. Intervention in fluency problems in pupils with Specific Language Impairment ( SLI ) / Intervención en problemas de fluidez en alumnado con Trastorno Específico del Lenguaje (TEL). Journal for the Study of Education and Development, 39:3, 466-498, DOI: $10.1080 / 02103702.2016 .1189118$

34.Ato E, Galián M, Cabello F. Intervención familiar en niños con trastornos del lenguaje: Una revisión. Electronic Journal of Research in Educational Psychology [Internet]. 2009;7(3):1419-1448.

35.Acosta V, Moreno Santana, A, Axpe Cabellero, Á. Intervención logopédica sobre habilidades narrativas en niños con Trastorno Específico del Lenguaje Speech and language intervention on 


Martínez, Caicedo, Rodríguez. Revista Científica Signos Fónicos, 2020, 6 (2): 42 - 61.
narrative skills in children with Specific Language Abstract. 2012;35(2):201-13.
Doi/Abs/10.1174/02103701280021803

36.Moreno A, Axpe Á, Acosta V. Intervención sobre la conciencia fonológica en sujetos con trastorno específico del lenguaje en contextos inclusivos: posibilidades y limitaciones. Universidad de la Lagua. 2011;63 (3):9-22.

37.Acosta V, Moreno A, Axpe Á. La detección e intervención en habilidades narrativas en niños con trastorno específico del lenguaje en contextos educativos. Universidad de la Lagua. 2017; 20(2), 387 404. DOI: 10.5944/educXX1.19053.

38.Acosta V. La intervención logopédica en los trastornos específicos del lenguaje. Rev Logop Foniatría y Audiol. 2012;32(2):67-74.

39. Beleño-Melo; Betsy-Patricia. Calidad de vida en la inclusión educativa de niños y adolescentes con discapacidad intelectual. Revistas Científica Signos Fónicos. 2019: 5 (1). 60-72, https://doi.org/10.24054/01204211.v1.n1.2019.3982

40.Maggiolo M, Pavez M, Coloma C. Terapia para el desarrollo narrativo en niños con trastorno específico del lenguaje. Rev Logop Foniatría y Audiol. 2003;23(2):98-108.

41.Manuscript, A., Approaches, E., \& Deconstructing, T. NIH Public Access. 2012, 40(6), 1301-1315. Doi.org/10.1007/s10439-011-0452-9.

42.González L. Trastorno específico del lenguaje (TEL): concepto y características. 2018;4(4):166-174. from: https://revistaselectronicas.ujaen.es/index.php/riai/article/download/4372/3586/

43.Acosta V, González N, Lorenzo C. Un análisis cualitativo de la estructura episódica, los recursos cohesivos y la diversidad léxica en la narrativa de alumnado con Trastorno Específico del Lenguaje. 2011;143-159.

44.Pera M, Canals A. Valoración dinámica de la habilidad narrativa: aplicación en un caso de trastorno específico de lenguaje. Rev Logop Foniatría y Audiol [Internet]. 2008;28(4):245-60.

45.Santana, A., Caballero, Á., \& Rodríguez, V. Del léxico y del procesamiento fonológico en escolares de educación infantil con trastorno específico del lenguaje. Revista de Investigación. 2012, 30(1): 7186. Doi.Org/10.6018/Rie.30.1.113861

46.Roqueta A, Estevan, R, Flores R. Cognición Social y competencia pragmática. El caso de los niños y niñas con Trastorno Específico del Lenguaje Social. International Journal of Psychological Research Lenguaje.2012, 5(51), 59-69.

47.Mantiñán N R, Badel M S, Fermoselle M S. Lenguaje y memoria de trabajo: implicancias en la detección e intervención del TEL.2014; 6(3), 47-54. Doi.Org/10.5579/Rnl.2014.0201

48.Moran M, Vera L, Morán M. Los trastornos del lenguaje y las Necesidades Educativas Especiales. Consideraciones para la atención en la escuela. Universidad y Sociedad. 2017;9(2):191-197.

49.Hincapié L, Giraldo M, Castro R, Lopera F, Pineda D, Lopera E. Propiedades lingüísticas de los trastornos específicos del desarrollo del lenguaje. Revista Latinoamericana de Psicología.2007, 39(1): 47-61. 
Martínez, Caicedo, Rodríguez. Revista Científica Signos Fónicos, 2020, 6 (2): 42 - 61.

50.Carballo G, Fresneda MD. Evaluación e intervención logopédica en el trastorno específico del lenguaje. 2005;41(1):73-82.

51.Acosta V. Algunos retos y propuestas en la conceptualización, evaluación e intervención del Trastorno Específico del Lenguaje (TEL) [Internet]. Revista Chilena de Fonoaudiología.2012;2636.DOI: $10.5354 / 0719-4692.2012 .24525$

52.Lum J, Conti G, Morgan A, Ullman M. Procedural learning deficits in specific language impairment (SLI): A meta-analysis of serial reaction time task performance. Córtex. 2014; 51(100): 1-10.

53.Acosta V, Ramírez G, Hernández S. Un estudio de la relación entre la memoria, la narrativa y el lenguaje en alumnado con trastorno específico del lenguaje (TEL). Universitas Psychologica. 2015; 14(2): 631-643.doi.org.10.11144/

54.Moreno J, García E; Guerrero E, Blázquez M. Competencia pragmática y adaptación psicosocial en niños sujetos a medidas de protección infantil. Salud Mental. 2010, 33(4): 333-340.

55.Mendoza E. La investigación actual en el Trastorno Específico del Lenguaje. Revista de Logopedia, Foniatría y Audiología. 2012; 32(2): 75-86.Doi.Org/10.1016/J.Rlfa.2012.03.001

56.Bázquez A. La pragmática en el aula de ele: la enseñanza de la cortesía verbal dirigida a estudiantes nativos de inglés marco ele. Revista de didáctica español lengua extranjera. 2015, 21(2): 1-54. Disponible en: https://www.redalyc.org/pdf/921/92152429001.pdf 